\title{
DIGITALLY PROGRAMMABLE PARTIALLY ACTIVE-R SINUSOIDAL OSCILLATORS
}

\author{
MUHAMMAD TAHER ABUELMA'ATTI, ABDULRAHMAN \\ KHALAF AL-ALI, SAMI SAUD BUHALIM AND \\ SYED TAUSEEF AHMED \\ King Fahd University of Petroleum and Minerals, Box 203, Dhahran 31261, Saudi Arabia
}

(Received March 2, 1994; in final form April 13, 1994)

New, simple sinusoidal oscillator circuits are proposed. Each circuit uses an internally compensated operational amplifier, a resistor, and a capacitor. The feasibility of obtaining digitally programmable sinusoidal oscillation is studied and a new digitally programmable capacitorless resistorless sinusoidal oscillator is developed.

\section{INTRODUCTION}

In active-RC circuits, it is highly desirable to have all the capacitors grounded [18]. This is attributed to several reasons. First, grounded capacitors are suitable for monolithic IC technology [9-11] and thin film fabrication [2]. In thin-film fabrication, if the capacitors are grounded, the etching process is eliminated and the number of contacts is reduced. Also, the parasitic capacitors surrounding the main capacitors can be easily accounted for or tuned out as they are now in parallel with the grounded capacitors [3]. Secondly, in CMOS technology, the use of grounded capacitors is an advantage as the bottom plate parasitic capacitor is eliminated altogether and the top plate parasitic capacitor can be accounted for easily as it becomes parallel to the main capacitor [12]. This explains the considerable interest in designing $\mathrm{RC}$ oscillators with grounded capacitors $[1,3,5,6,13]$.

In a recent publication, Senani [12] presents a grounded-capacitor sinusoidal oscillator using operational amplifier compensation poles. This circuit can provide voltage-controlled oscillation by employing three operational amplifiers; at least two of them are internally compensated, one resistor, a grounded capacitor, and two dc power supplies; at least one of them is variable.

The purpose of this paper is to show that a simple digitally programmable voltage(current)-controlled oscillator can be implemented using a grounded capacitor, an internally compensated operational amplifier, two operational transconductance amplifiers (OTAs), and only one fixed dc power supply. 


\section{PROPOSED CIRCUIT}

The new proposed circuit is shown in Fig. 1a and its equivalent circuit is shown in Fig. 1b with [14]

$R=\frac{1}{g_{m}}$

$g_{m}=\frac{I_{A B C}}{2 V_{T}}$

where $V_{T}=26 \mathrm{mV}$ is the thermal voltage at room temperature and $I_{A B C}$ is the auxiliary bias current of the OTAs. Assuming that the operational amplifier is characterized by

$G=\frac{G_{o} \omega_{a} \omega_{b}}{\left(s+\omega_{a}\right)\left(s+\omega_{b}\right)}$

routine analysis shows that the characteristic equation of the circuit of Fig. 1a is given by

$\alpha G_{o} \omega_{a} \omega_{b}+\left(\omega_{a}+s\right)\left(\omega_{b}+s\right)(1+s \tau)=0$

where

$\tau=C R_{o} / /\left(R+R_{\text {inp }}\right)$

$\alpha=R_{\text {inp }} /\left(R_{\text {inp }}+R+R_{o}\right), R_{o}$ is the output resistance of the operational amplifier, and $R_{\text {inp }}$ is the differential input resistance of the operational amplifier. The frequency of oscillation and the condition of oscillation are, therefore, given by

$\omega_{o}^{2}=\omega_{a} \omega_{b}+\frac{\omega_{a}+\omega_{b}}{\tau}$

and

$G_{o}=\frac{1}{\alpha}\left(1+\frac{1}{\tau \omega_{a}}+\frac{1}{\tau \omega_{b}}\right) \frac{1}{1+\tau\left(\omega_{a}+\omega_{b}\right)}$

The condition of (7) can be easily satisfied in practical operational amplifiers.

From (1) and (6) it follows that the frequency of oscillation can be expressed as

$\omega_{o}^{2}=\omega_{a} \omega_{b}+\frac{\omega_{a}+\omega_{b}}{C R_{o} / /\left(R_{\text {inp }}+2 V_{T} / I_{A B C}\right)}$ 


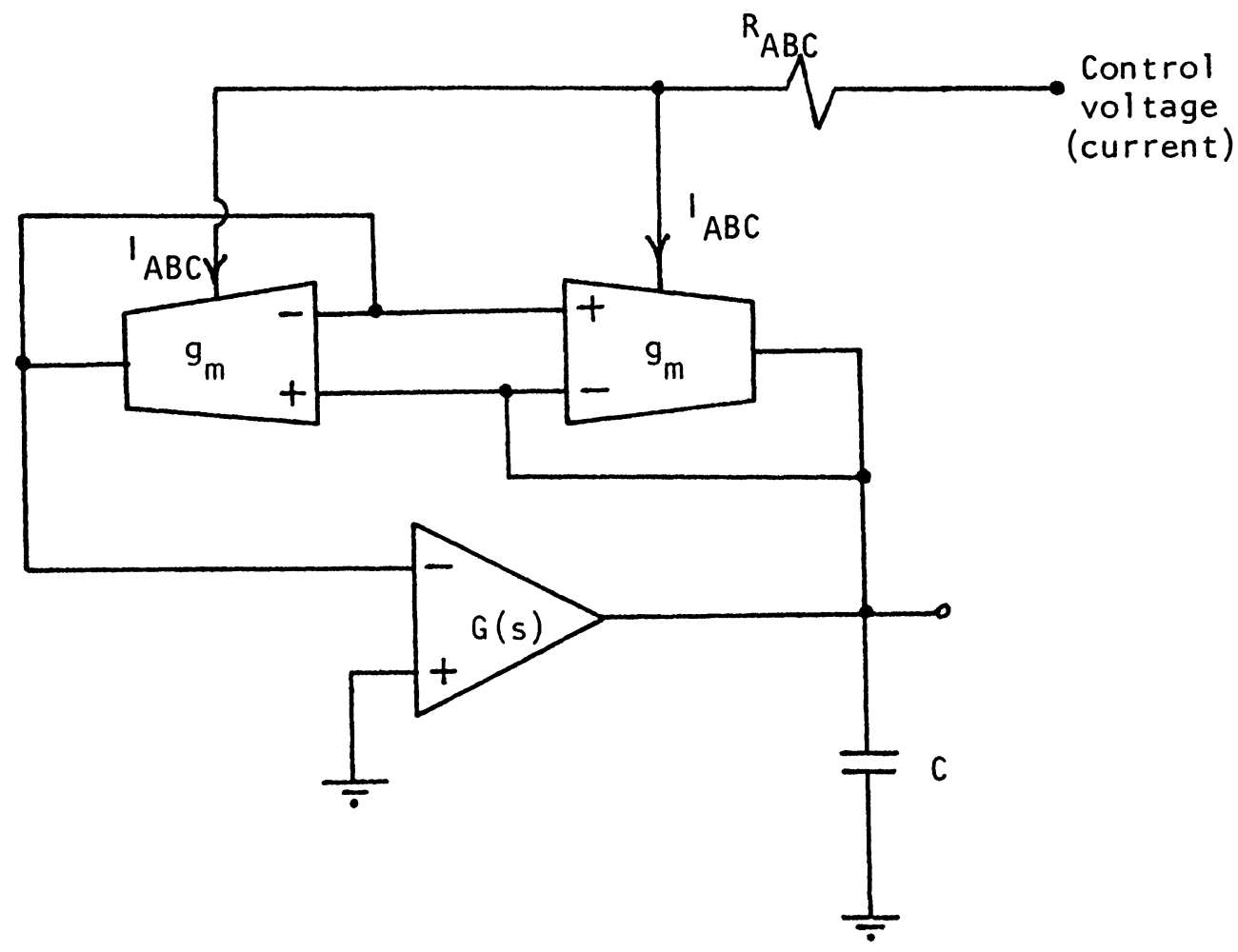

(a)

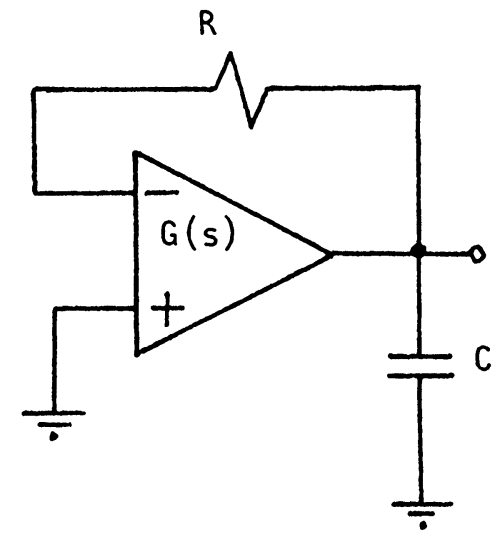

(b)

FIGURE 1 Proposed oscillator circuit (a) and its equivalent circuit (b) 
From (8) it is obvious that the frequency of oscillation can be tuned by adjusting the auxiliary bias current $I_{A B C}$ of the OTAs and, thus, by obtaining this current from the output of a digital-to-analog converter (DAC), the realization of a digitally programmable tunable oscillator is feasible.

An alternative oscillator circuit, shown in Fig. 2a with its equivalent circuit shown in Fig. $b$, can be obtained by moving the capacitor $C$ from the output terminal of the operational amplifier to the inverting input terminal. Assuming that the operational amplifier is characterized by the two-pole model of (2), routine analysis shows that the characteristic equation of this circuit is given by [15]

$$
(1+s C R)\left(\omega_{a} \omega_{b}+s\left(\omega_{a}+\omega_{b}\right)+s^{2}\right)+G_{o} \omega_{a} \omega_{b}=0
$$

The frequency of oscillation and the condition of oscillation are, therefore, given by

$\omega_{o}^{2}=\omega_{a} \omega_{b}+\frac{\omega_{a}+\omega_{b}}{R C}$

and

$G_{o}=\frac{\omega_{b}}{\omega_{a}}+\frac{1}{\omega_{a} C R}+\omega_{b} C R$

The condition of (11) can be easily satisfied in practical operational amplifiers.

From (1) and (10) it follows that the frequency of oscillation can be expressed as

$\omega_{o}^{2}=\omega_{a} \omega_{b}+\frac{\omega_{a} \omega_{b}}{C} \frac{I_{A B C}}{2 V_{T}}$

From (12) it is obvious that the frequency of oscillation can be tuned by adjusting the auxiliary bias current $I_{A B C}$ of the OTAs. By obtaining this current from the output of a digital-to-analog converter (DAC), the realization of a digitally programmable electronically tunable oscillator is feasible. It is worthwhile mentioning that a capacitorless oscillator circuit can be obtained from the circuit of Fig. 2. This can be achieved by exploiting the differential input capacitance of the operational amplifier to advantage rather than connecting an external capacitance $\mathrm{C}$.

\section{EXPERIMENTAL RESULTS}

The oscillator circuit of Fig. 1 was built and tested using the operational amplifier 741 , the OTA 3080 , and an externally connected capacitor $0.01 \mu F$. Variation of the frequency of oscillation with the control voltage of the operational transconductance amplifier is shown in Fig. 3. When the control voltage was varied from $-4 \mathrm{~V}$ to $7 \mathrm{~V}$, the frequency of oscillation varied from $87 \mathrm{KHz}$ to $416 \mathrm{KHz}$. The 


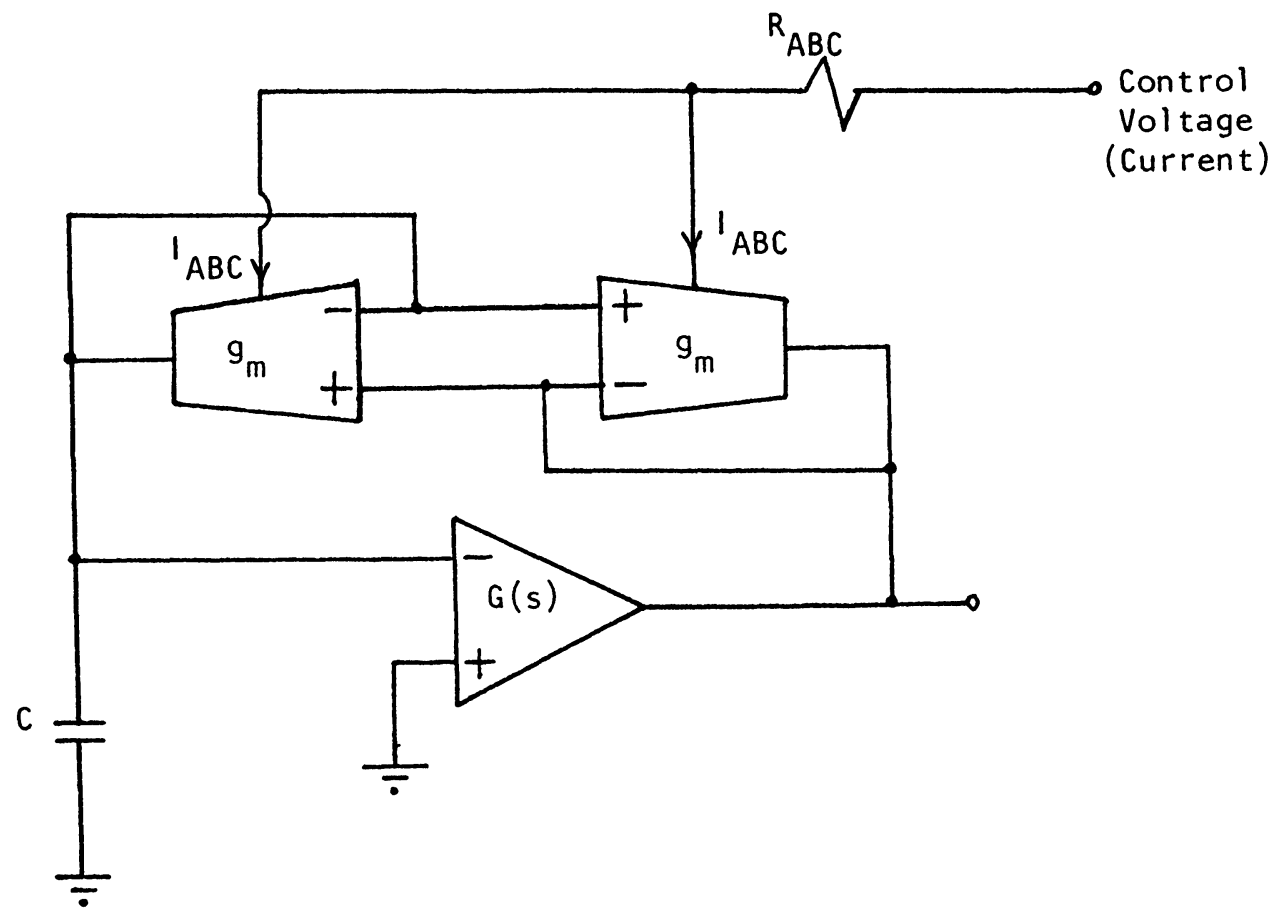

(a)

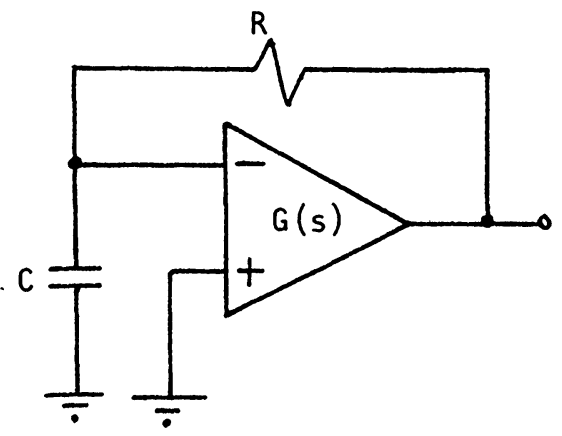

(b)

FIGURE 2 Another oscillator circuit (a) and its equivalent circuit (b) [15]

oscillator circuit of Fig. 3 was also built and tested using the operational amplifier 741, the operational transconductance amplifier 3080, and an external capacitor $0.01 \mu F$. Variation of the frequency of oscillation with the control voltage of the operational transconductance amplifier is shown in Fig. 3. When the control voltage was varied from $-4 \mathrm{~V}$ to $7 \mathrm{~V}$, the frequency of oscillation varied from $10 \mathrm{KHz}$ to 


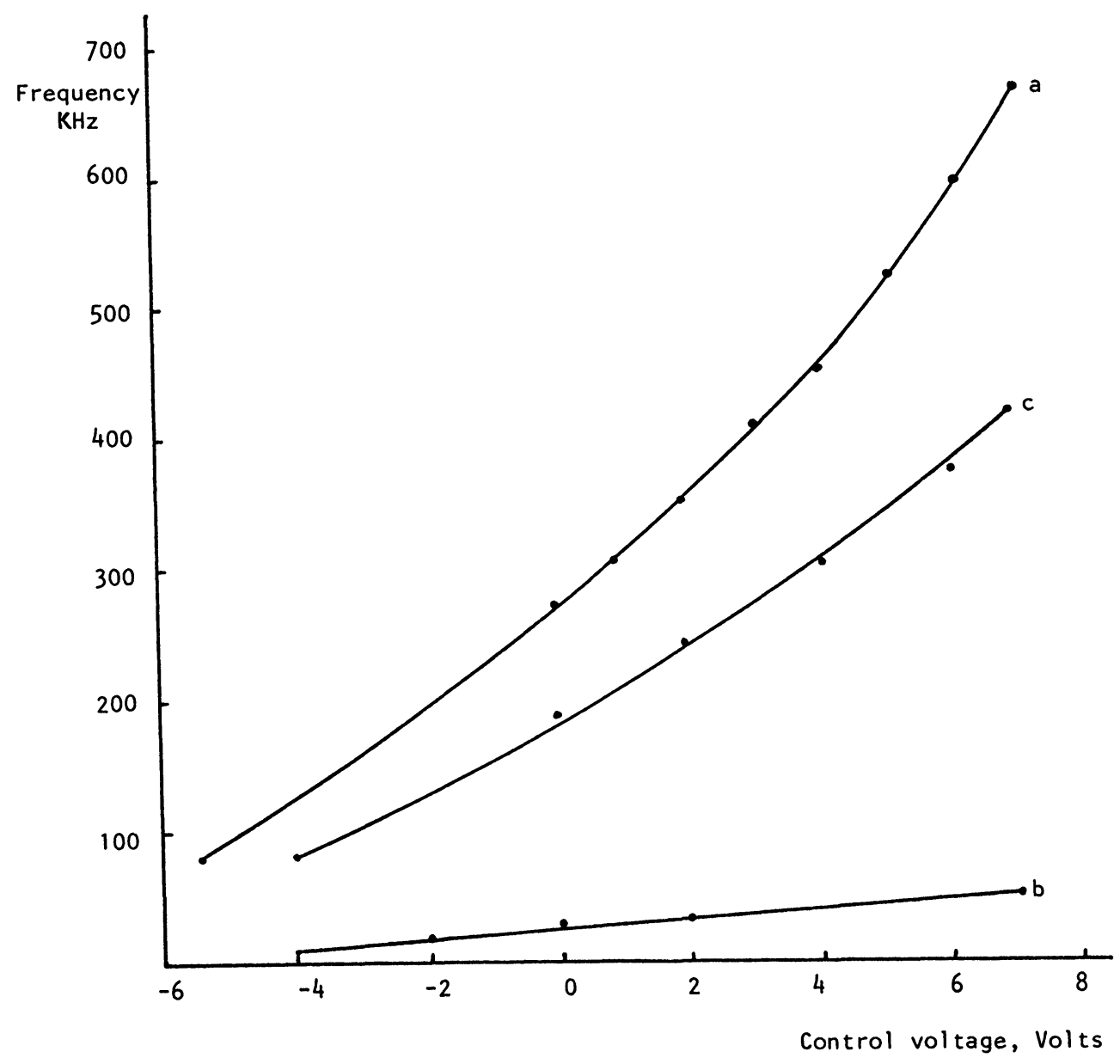

FIGURE 3 Variation of the frequency of oscillation with the control voltage for: (a) Circuit of Fig. 2 without external capacitor, but with cable and oscilloscope capacitances; (b) Circuit of Fig. 2 with external capacitor $=0.01 \mathrm{uF}$; (c) Circuit of Fig. 1 with external capacitor $=0.01 \mathrm{uF}$. In all cases $R_{A B C}$ $=400 \mathrm{~K}$

$52 \mathrm{KHz}$. The operation of the circuit was also tested with no external capacitor added other than the oscilloscope and cable capacitances, which may be about 120 $\mathrm{pF}$. Variation of the frequency of oscillation with control voltage of the operational transconductance amplifier is shown in Fig. 3. When the control voltage was varied from $-5.5 \mathrm{~V}$ to $7 \mathrm{~V}$, the frequency of oscillation varied from $86 \mathrm{KHz}$ to $666 \mathrm{KHz}$. By obtaining this control voltage from the output of a digital-to-analog converter, the frequency of oscillation was varied from $100 \mathrm{KHz}$ to $239 \mathrm{KHz}$ when the hexadecimal input varied from 60 to $\mathrm{E} 0$.

\section{CONCLUSION}

In this paper, new digitally programmable partially active-R oscillator circuits, using the operational amplifier poles, have been presented. It has been shown that by 
exploiting the differential input capacitance of the operational amplifier, a capacitorless, resistorless, sinusoidal oscillator can be obtained using only an operational amplifier and two operational transconductance amplifiers. For stable operation of the proposed circuits, the operational amplifier parameters $G_{o}, \omega_{a}$, and $\omega_{b}$ must be stabilized. Therefore, we recommend a temperature compensated operational amplifier such as the LM324. Moreover, since $V_{T}$ is involved in the expression of the frequency of oscillation, temperature compensation of the operational transconductance amplifiers is recommended particularly if the circuit is to be used under varying environmental conditions. This can be easily achieved using readily available techniques [16].

\section{REFERENCES}

1. R.S. Sidorowicz, "An abundance of sinusoidal RC oscillators," Proceedings IEEE, Vol. 119, 1972, pp. 283-293

2. M.S. Abougabal, B.B. Battacharyya and M.N.S. Swamy, "An optimal design of RC active filters using grounded capacitors," International Journal of Circuit Theory and Applications, Vol. 6, 1978, pp. 31-40

3. R.S. Sidorowicz, "Some novel RC oscillators for radio frequencies," Electronic Engineering, Vol. 39,1967, pp. 498-502, 560-564

4. L.P. Huelsman, "Equal-valued-capacitor active RC network realisation of a third-order lowpass Butterworth characteristic," Electronics Letters, Vol. 7, 1971, pp. 271-271

5. M. Sundramurthy, B.B. Battacharyya and M.N.S. Swamy, "A simple voltage controlled oscillator with grounded capacitors," Proceedings IEEE, Vol. 65, 1977, pp. 1612-1614

6. B.B. Battacharyya, M. Sundramurthy and M.N.S. Swamy, "Realisation of tunable RC-active oscillators using grounded capacitors and voltage amplifiers," International Journal of Circuit Theory and Applications," Vol. 8, 1980, pp. 355-371

7. R.A. Stein, "Synthesis of grounded-capacitor multiport RC networks," IEEE International Symposium on Circuit Theory, 1971, pp. 49-50

8. M.S. Abougabal and B.B. Battacharyya, "Easily integrable very-low-sensitivity active RC filter," Electronics Letters, Vol. 8, 1972, pp. 303-304

9. G.S. Moschytz and W. Thelen, "Design of hybrid integrated-filter building blocks," IEEE Journal of Solid State Circuits, Vol. SC-5, 1970, pp. 99-107

10. G.S. Moschytz, "Gain-sensitivity product-a figure of merit for hybrid-integrated filters using single operational amplifier," IEEE Journal of Solid State Circuits, Vol. SC-6, 1971, pp. 103-110

11. R.W. Newcomb, "Active integrated synthesis," Prentice-Hall, New Jersey, 1968

12. R. Senani, "Simple sinusoidal oscillator using OpAmp compensation poles," Electronics Letters, Vol. 29, 1993, pp. 452-453

13. M.T. Darkani and B.B. Bhattacharyya, "Generation and design of canonic grounded-capacitor variable-frequency RC-active oscillators," IEEE Proceedings, Vol. 132, Part G, 1985, pp. 153160

14. I.A. Khan and M.T. Ahmed, "Realisation of tunable floating resistors," Electronics Letters, Vol. 22,1986 , pp. $799-800$

15. M.T. Abuelma'atti and W.A. Almansoury, "Two novel oscillators," Electronics and Wireless World, Vol. 92, \#1611, Jan. 1987, pp. 67-68.

16. M.T. Ahmed, M.R. Khan and T. Altaf, "A simple temperature stabilization scheme for monolithic operational transconductance amplifiers," Modelling, Simulation and Control, A, Vol. 25, \#2, 1989, pp. 49-56. 

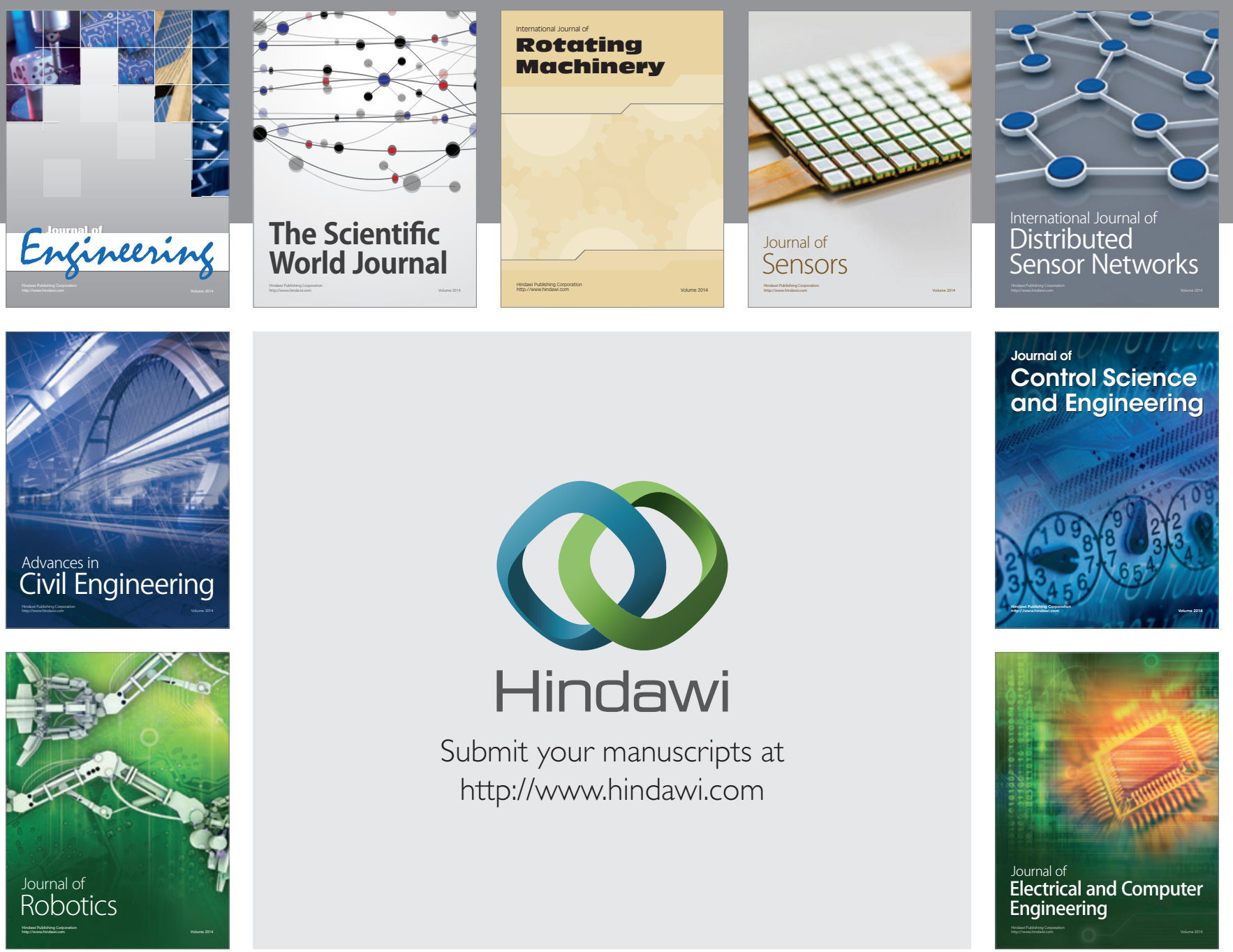

Submit your manuscripts at

http://www.hindawi.com
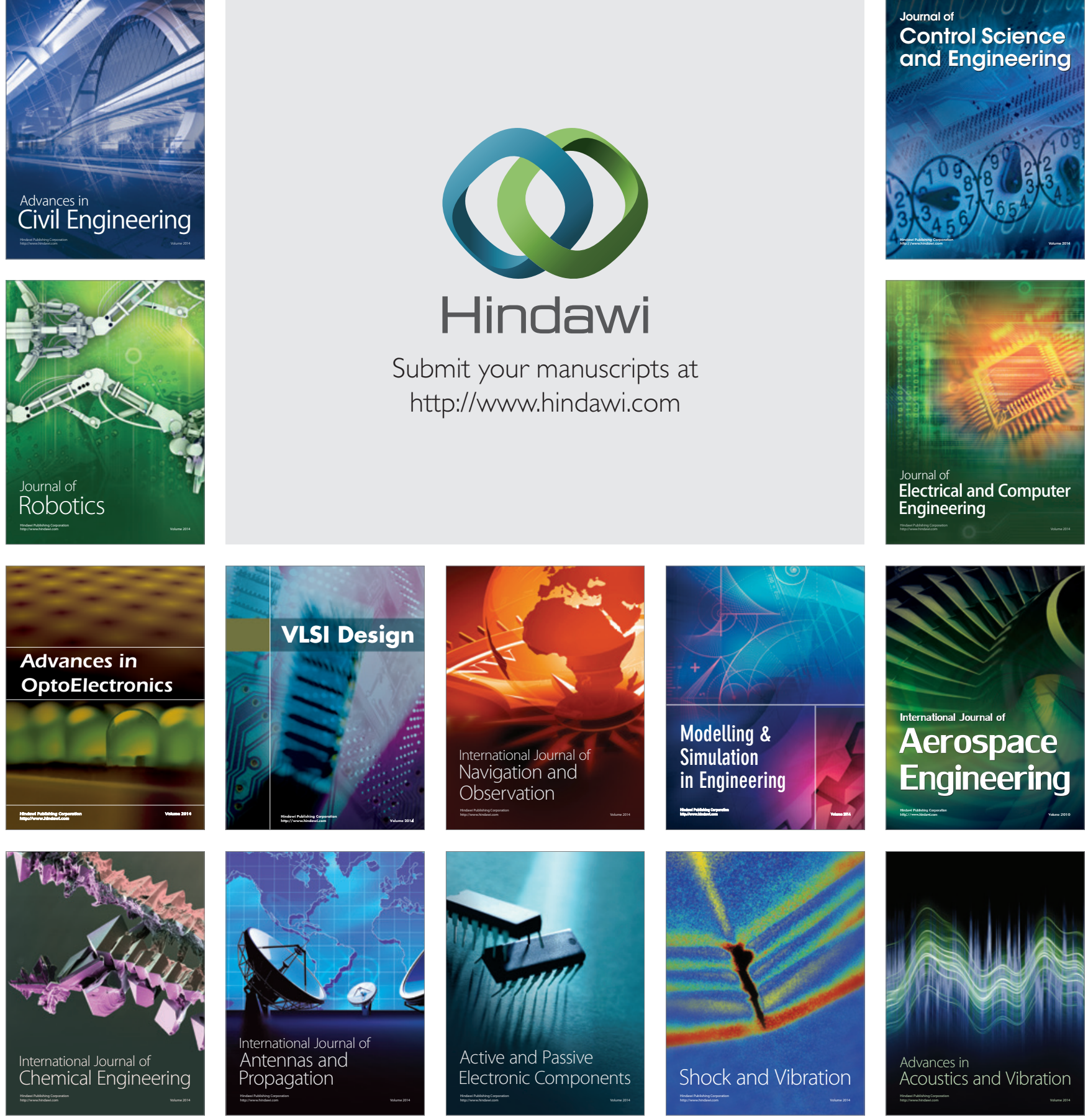